\title{
MALLIAVIN DIFFERENTIABILITY OF THE HESTON VOLATILITY AND APPLICATIONS TO OPTION PRICING
}

\author{
ELISA ALÒS, * University Pompeu Fabra Barcelona \\ CHRISTIAN-OLIVER EWALD, ${ }^{* *}$ University of St Andrews
}

\begin{abstract}
We prove that the Heston volatility is Malliavin differentiable under the classical Novikov condition and give an explicit expression for the derivative. This result guarantees the applicability of Malliavin calculus in the framework of the Heston stochastic volatility model. Furthermore, we derive conditions on the parameters which assure the existence of the second Malliavin derivative of the Heston volatility. This allows us to apply recent results of Alòs (2006) in order to derive approximate option pricing formulae in the context of the Heston model. Numerical results are given.
\end{abstract}

Keywords: Malliavin calculus; stochastic volatility model; Heston model; Cox-IngersollRoss process; Hull and White formula; option pricing

2000 Mathematics Subject Classification: Primary 91B28

Secondary $60 \mathrm{H} 07 ; 60 \mathrm{H} 10 ; 60 \mathrm{H} 30$

\section{Introduction}

In recent years Malliavin calculus has appeared as a major tool in both theoretical and computational mathematical finance. This fact is documented by the large number of published articles in this area. The assumptions on the possibly multidimensional diffusion process $X_{t}$, which determines the factors of the model, in general require as a minimal condition that the coefficient functions $\beta$ and $\sigma$ in

$$
\mathrm{d} X_{t}=\beta\left(X_{t}, t\right) \mathrm{d} t+\sigma\left(X_{t}, t\right) \mathrm{d} \mathbb{W}_{t},
$$

where $\mathbb{W}_{t}$ denotes a standard Brownian motion, are continuously differentiable and satisfy a global Lipschitz condition. These assumptions work fine with the standard Black-Scholes model or the more general models based on linear stochastic differential equations (SDEs). Problems occur, however, when one uses more advanced models, like the Heston stochastic volatility model. In this model the stock price is given by the equation

$$
\mathrm{d} S_{t}=S_{t}\left(b \mathrm{~d} t+\sqrt{v_{t}} \mathrm{~d} B_{t}\right),
$$

where $b$ denotes a real constant and $B_{t}$ denotes a Brownian motion, but in contrast to the standard Black-Scholes model, the volatility $v_{t}$ is itself a diffusion process satisfying the stochastic differential equation

$$
\mathrm{d} v_{t}=\kappa\left(\theta-v_{t}\right) \mathrm{d} t+v \sqrt{v_{t}} \mathrm{~d} W_{t}
$$

Received 4 September 2006; revision received 21 December 2007.

* Postal address: Department of Economics, University Pompeu Fabra Barcelona, c/Ramon Trias fargas, 25-27, 08005 Barcelona, Spain. Email address: elisa.alos@upf.edu

** Postal address: Department of Economics, University of St Andrews, St Salvator's College, St Andrews, Fife KY16 9AL, UK. Email address: c.o.ewald@st-andrews.ac.uk 
where $W_{t}$ denotes a possibly correlated second Brownian motion and $\kappa, \theta$, and $\nu$ are positive constants. Obviously, the coefficient functions of this model do not satisfy the standard assumptions. The square root function is neither differentiable at 0 nor globally Lipschitz. In this article we present a direct proof of the Malliavin differentiability of the Heston volatility and its square root and give explicit expressions for their derivatives. Furthermore, we discuss the existence of the second Malliavin derivative and derive conditions on the parameters $\kappa, \theta$, and $v$ which guarantee its existence. Recently, in [1] Malliavin calculus techniques have been applied in order to obtain an extension of the classical Hull and White formula (see [9]) for the case of correlated stock and volatility. In order to apply the results to the Heston model, Malliavin differentiability as well as certain integrability conditions of the Malliavin derivative of the Heston volatility have to be verified. Our application includes an adaptation of the results of [1] to the case of the Heston volatility, a derivation of a new approximative option pricing formula for the Heston model, and a precise analysis of the accuracy of this approximation.

The structure of the article is as follows. In Section 2 we give an explicit approximating sequence for the Heston volatility, while in Section 3 we provide some preliminaries on Malliavin calculus. We study the Malliavin differentiability of the Heston volatility in Section 4 and present our two main theoretical results. In Section 5 we include our application followed by numerical results in Section 6. The main conclusions are summarized in Section 7.

\section{The Heston volatility model and an approximating sequence}

As mentioned in the introduction, the Heston stochastic volatility model consists of a money market account which we do not specify at the moment, a stock $S_{t}$, and the volatility process $v_{t}$ with dynamics specified in (1.1) and (1.2), where it is assumed that $\kappa, \theta$, and $v$ are positive constants; see [8]. In the following we consider one fixed probability space $(\Omega, \mathcal{G}, \mathrm{P})$ on which there is defined a Brownian motion $\left(W_{t}\right)$ that is filtered by the augmented and completed Brownian filtration which we denote with $\left(g_{t}\right)$. We also fix an interval $[0, T]$. A standard assumption when using the Heston model is that $2 \kappa \theta \geq v^{2}$. This is often called Novikov's condition. Given that $v_{0}>0$, this condition guarantees that the volatility process is always positive, i.e. $\mathrm{P}\left(\left\{v_{t}>0\right.\right.$ for all $\left.\left.t>0\right\}\right)=1$. We assume that $v_{0}>0$ and that Novikov's condition holds. It is then possible to consider the square root process $\sigma_{t}:=\sqrt{v_{t}}$. It follows from the Itô formula that this process satisfies

$$
\mathrm{d} \sigma_{t}=\left(\left(\frac{\kappa \theta}{2}-\frac{v^{2}}{8}\right) \frac{1}{\sigma_{t}}-\frac{\kappa}{2} \sigma_{t}\right) \mathrm{d} t+\frac{v}{2} \mathrm{~d} W_{t} .
$$

We note that Novikov's condition implies, in particular, that the factor $\left(\kappa \theta / 2-v^{2} / 8\right)$ appearing in the drift term of $\sigma_{t}$ is positive. This will play a significant role later. It is not a priori clear that the SDE (2.1) admits a unique strong solution, but the Yamada-Watanabe lemma [11, Chapter 5, Proposition 2.18] obviously implies uniqueness of the solution of the SDE (1.2). For any solution $\sigma_{t}$ of the $\operatorname{SDE}(2.1)$, by applying the Itô formula we find that $\sigma_{t}^{2}$ is a solution of the SDE (1.2). As the latter one is unique, we conclude uniqueness of the solution for the SDE (2.1) up to a sign. However, if $\sigma_{t}$ solves (2.1), it is obvious that $-\sigma_{t}$ does not and, therefore, we find uniqueness of the solution of the SDE (2.1). In order to show in Section 4 that $\sigma_{t}$ is Malliavin differentiable we will now define an approximating sequence. Let $\varepsilon>0$, and let $\Phi_{\varepsilon}(x)$ be a continuously differentiable function satisfying $\Phi_{\varepsilon}(x)=1$ if $x \geq 2 \varepsilon$ and $\Phi_{\varepsilon}(x)=0$ if $x<\varepsilon$, while $\Phi_{\varepsilon}(x) \leq 1$ for all $x \in \mathbb{R}$. We note that in this case the derivative $\Phi_{\varepsilon}^{\prime}(x)=0$ if $x<\varepsilon$ or $x \geq 2 \varepsilon$. Furthermore, we define the function $\Lambda_{\varepsilon}(x)=\Phi_{\varepsilon}(x)(1 / x)$ 
with $\Lambda_{\varepsilon}(0)=0$. The function $\Lambda_{\varepsilon}(x)$ is bounded and continuously differentiable satisfying $\Lambda_{\varepsilon}^{\prime}(x)=\Phi_{\varepsilon}^{\prime}(x)(1 / x)-\Phi_{\varepsilon}(x)\left(1 / x^{2}\right)$. In particular, $\Lambda_{\varepsilon}^{\prime}(x)=-1 / x^{2}$ if $x \geq 2 \varepsilon$ and $\Lambda_{\varepsilon}^{\prime}(x)=0$ if $x<\varepsilon$. Let us now define our approximations $\sigma_{t}^{\varepsilon}$ as the solutions of the SDEs

$$
\mathrm{d} \sigma_{t}^{\varepsilon}=\left(\left(\frac{\kappa \theta}{2}-\frac{\nu^{2}}{8}\right) \Lambda_{\varepsilon}\left(\sigma_{t}^{\varepsilon}\right)-\frac{\kappa}{2} \sigma_{t}^{\varepsilon}\right) \mathrm{d} t+\frac{v}{2} \mathrm{~d} W_{t},
$$

with $\sigma_{0}^{\varepsilon}=\sigma_{0}$ for all $\varepsilon>0$.

Proposition 2.1. For each $t \in[0, T]$, the sequence $\sigma_{t}^{\varepsilon}$ converges to $\sigma_{t}$ in $L^{2}(\Omega)$.

Proof. We use the dominated convergence theorem in order to obtain this result. Let us first prove that $\sigma_{t}^{\varepsilon}$ converges to $\sigma_{t}$ pointwise. This follows from a standard localization argument. For each $\varepsilon>0$, define a stopping time $\tau_{\varepsilon}$ via $\tau_{\varepsilon}(\omega):=\inf \left\{t \mid \sigma_{t}(\omega) \leq \varepsilon\right\}$. Letting $\varepsilon$ go to 0 , the sequence of $\left(\tau_{\varepsilon}\right)$ defines an increasing sequence of stopping times, and it follows from the strict positivity of $\sigma_{t}$ that $\lim _{\varepsilon \rightarrow 0} \tau_{\varepsilon}=\infty$ almost surely (a.s.). Denoting by $\sigma^{\tau_{\varepsilon}}$ the process obtained from $\sigma$ by stopping at $\tau_{\varepsilon}$, it then follows, from the choice of the function $\Lambda_{\varepsilon}(x),(2.1)$, and (2.2), that $\sigma_{t}^{\tau_{2 \varepsilon}}=\sigma_{t}^{\varepsilon}$ for all $t \leq \tau^{\varepsilon}$. Now, for fixed $t \in[0, T]$, letting $\varepsilon$ go to 0 , we obtain $\lim _{\varepsilon \rightarrow 0} \sigma_{t}^{\varepsilon}=\lim _{\varepsilon \rightarrow 0} \sigma_{t}^{\tau_{2 \varepsilon}}=\sigma_{t}$ a.s. Let us now prove that, for each $t \in[0, T], \sigma_{t}^{\varepsilon}$ converges to $\sigma_{t}$ in $L^{2}(\Omega)$. For this, let us consider the Ornstein-Uhlenbeck process $u_{t}$ satisfying $u_{0}=\sigma_{0}$ and

$$
\mathrm{d} u_{t}=-\frac{\kappa}{2} u_{t} \mathrm{~d} t+\frac{v}{2} \mathrm{~d} W_{t}
$$

We show that $u_{t} \leq \sigma_{t}^{\varepsilon} \leq \sigma_{t}$ for each $t \in[0, T]$ a.s. The first inequality follows directly from the Yamada-Watanabe comparison lemma. To prove the second inequality, this lemma cannot be applied directly as the drift term in the SDE for $\sigma_{t}$ is not continuous. Since we know, however, that under our assumptions on the coefficients $\sigma_{t}>0$ a.s., the second inequality would indeed follow from $\left(\sigma_{t}^{\varepsilon}\right)^{2} \leq \sigma_{t}^{2}$. In fact, applying Itô's formula to $v_{t}^{\varepsilon}=\left(\sigma_{t}^{\varepsilon}\right)^{2}$ gives

$$
\mathrm{d} v_{t}^{\varepsilon}=\left(\left(\kappa \theta-\frac{v^{2}}{4}\right) \sqrt{v_{t}^{\varepsilon}} \Lambda_{\epsilon}\left(\sqrt{v_{t}^{\varepsilon}}\right)-\kappa v_{t}^{\varepsilon}+\frac{v^{2}}{4}\right) \mathrm{d} t+v \sqrt{v_{t}^{\varepsilon}} \mathrm{d} W_{t},
$$

while $v_{t}=\sigma_{t}^{2}$ satisfies (1.2). For both $v_{t}^{\varepsilon}$ and $v_{t}$, the condition on the diffusion coefficient in [11, Chapter 5, Proposition 2.18] can be verified easily by choosing the function $h(x)=v \sqrt{x}$. Obviously, the drift term in (1.2) is globally Lipschitz. In addition, it is not hard to verify that the drift term corresponding to $v_{t}^{\varepsilon}$ is globally Lipschitz. Therefore, we can conclude the second inequality from

$$
\begin{array}{rlrl} 
& & \left(\kappa \theta-\frac{v^{2}}{4}\right) \sqrt{x} \Lambda_{\epsilon}(\sqrt{x}) & -\kappa x+\frac{v^{2}}{4} \leq \kappa(\theta-x), \\
& \Longleftrightarrow \quad \kappa \theta\left(\sqrt{x} \Lambda_{\epsilon}(\sqrt{x})-1\right) & \leq \frac{v^{2}}{4}\left(\sqrt{x} \Lambda_{\epsilon}(\sqrt{x})-1\right), \\
& \Leftrightarrow & \kappa \theta & \geq \frac{v^{2}}{4},
\end{array}
$$

the latter being true owing to Novikov's condition. For the last equivalence, we used the inequality $0 \leq \sqrt{x} \Lambda_{\epsilon}(\sqrt{x}) \leq 1$. Now it follows from $u_{t} \leq \sigma_{t}^{\varepsilon} \leq \sigma_{t}$ that $\left|\sigma_{t}^{\varepsilon}\right| \leq\left|u_{t}\right|+\left|\sigma_{t}\right|$. Since, obviously, $u_{t}$ and $\sigma_{t}$ belong to $L^{2}(\Omega)$, the dominated convergence theorem implies the desired convergence. 


\section{A short review of Malliavin calculus}

Let us review some of the basic features of Malliavin calculus. A standard reference for this is [12]. Let us consider the set $\&$ of cylindrical functionals $F: \Omega \rightarrow \mathbb{R}$, given by $F=$ $f\left(W_{t_{1}}, \ldots, W_{t_{l}}\right)$, where $f \in C_{b}^{\infty}\left(\mathbb{R}^{l}\right)$ is a smooth function with bounded derivatives of all orders and $W_{t}$ denotes a Brownian motion on $\Omega$. We define the Malliavin derivative operator on $\&$ via

$$
D_{s} F:=\sum_{i=1}^{l} \frac{\partial f}{\partial x_{i}}\left(W_{t_{1}}(\omega), \ldots, W_{t_{l}}(\omega)\right) \mathbf{1}_{\left[0, t_{i}\right]}(s) .
$$

This operator and the iterated operators $D^{n}$ are closable and unbounded from $L^{p}(\Omega)$ into $L^{p}\left(\Omega \times[0, T]^{n}\right)$ for all $n \geq 1$. Their respective domains are denoted by $\mathbb{D}^{n, p}$ and are obtained as the closure of $\&$ with respect to the norms defined by

$$
\|F\|_{n, p}^{p}=\|F\|_{L^{p}(\Omega)}^{p}+\sum_{k=1}^{n}\left\|D^{k} F\right\|_{L^{p}\left(\Omega \times[0, T]^{k}\right)}^{p} .
$$

The adjoint of the Malliavin derivative operator $D: \mathbb{D}^{1,2} \rightarrow L^{2}(\Omega \times[0, T])$ is called the Skorohod integral and denoted by $\delta$. This operator has the property that its domain contains the class $L_{a}^{2}(\Omega \times[0, T])$ of square integrable adapted stochastic processes and its restriction to this class coincides with the Itô integral. We will make use of the notation $\delta(u)=\int_{0}^{T} u_{t} \mathrm{~d} W_{t}$, and recall that $\mathbb{L}^{n, 2}:=L^{2}\left([0, T], \mathbb{D}^{n, 2}\right)$ is included in the domain of $\delta$ for all $n \geq 1$. For more details, we refer the reader to [12, Chapter 1]. Later, we will use the following anticipative Itô formula; see [2].

Proposition 3.1. Let us consider the processes $X_{t}=X_{0}+\int_{0}^{t} u_{s} \mathrm{~d} W_{s}+\int_{0}^{t} v_{s} \mathrm{~d}$ s, where $X_{0}$ is $\mathcal{F}_{0}$-measurable and $u, v \in L_{a}^{2}([0, T] \times \Omega)$. Furthermore, consider a process $Z_{t}=\int_{t}^{T} \theta_{s} \mathrm{~d} s$ for some $\theta \in \mathbb{L}^{1,2}$. Let $F: \mathbb{R}^{3} \rightarrow \mathbb{R}$ be a twice continuously differentiable function for which there exists a positive constant $C$ such that, for all $t \in[0, T], F$ and its derivatives evaluated in $\left(t, X_{t}, Z_{t}\right)$ are bounded by $C$. Then it follows that

$$
\begin{aligned}
F\left(t, X_{t}, Z_{t}\right)= & F\left(0, X_{0}, Z_{0}\right)+\int_{0}^{t} \frac{\partial F}{\partial s}\left(s, X_{s}, Z_{s}\right) \mathrm{d} s+\int_{0}^{t} \frac{\partial F}{\partial x}\left(s, X_{s}, Z_{s}\right) \mathrm{d} X_{s} \\
& +\int_{0}^{t} \frac{\partial F}{\partial z}\left(s, X_{s}, Z_{s}\right) \mathrm{d} Z_{s}+\int_{0}^{t} \frac{\partial^{2} F}{\partial x \partial z}\left(s, X_{s}, Z_{s}\right)\left(\int_{s}^{T} D_{s} \theta_{r} \mathrm{~d} r\right) u_{s} \mathrm{~d} s \\
& +\frac{1}{2} \int_{0}^{t} \frac{\partial^{2} F}{\partial x^{2}}\left(s, X_{s}, Z_{s}\right) u_{s}^{2} \mathrm{~d} s
\end{aligned}
$$

\section{Malliavin differentiability of the Heston volatility}

In this section we will show that both the Heston volatility, $v_{t}$, as well as its square root $\sigma_{t}$ belong to $\mathbb{D}^{1,2}$. We will also derive conditions under which the second Malliavin derivative of the Heston volatility exists.

Lemma 4.1. We have $\sigma_{t}^{\varepsilon} \in \mathbb{D}^{1,2}$ and, for $r<t$,

$$
D_{r} \sigma_{t}^{\varepsilon}=\frac{v}{2} \exp \left(\int_{r}^{t}\left(-\frac{\kappa}{2}+\left(\frac{\kappa \theta}{2}-\frac{\nu^{2}}{8}\right) \Lambda_{\varepsilon}^{\prime}\left(\sigma_{s}^{\varepsilon}\right)\right) \mathrm{d} s\right) .
$$


Proof. Follows directly from [6, Theorem 2.1].

We are now ready to prove the following result.

Proposition 4.1. Assuming that $2 \kappa \theta \geq v^{2}$, we have $\sigma \in \mathbb{D}^{1,2}$ and, for $r<t$,

$$
D_{r} \sigma_{t}=\frac{v}{2} \exp \left(\int_{r}^{t}\left(-\frac{\kappa}{2}-\left(\frac{\kappa \theta}{2}-\frac{v^{2}}{8}\right) \frac{1}{\sigma_{t}^{2}}\right) \mathrm{d} s\right) .
$$

Proof. We know from Proposition 2.1 that, for each $t \in[0, T]$, the sequence $\sigma_{t}^{\varepsilon}$ converges to $\sigma_{t}$ in $L^{2}(\Omega)$. Since this convergence is also pointwise, we conclude, by using the properties of the function $\Lambda_{\varepsilon}(x)$, that

$$
D_{r} \sigma_{t}^{\varepsilon}=\frac{v}{2} \exp \left(\int_{r}^{t}\left(-\frac{\kappa}{2}-\left(\frac{\kappa \theta}{2}-\frac{v^{2}}{8}\right) \Lambda_{\varepsilon}^{\prime}\left(\sigma_{t}^{\varepsilon}\right)\right) \mathrm{d} s\right)
$$

converges pointwise to

$$
G:=\frac{v}{2} \exp \left(\int_{r}^{t}\left(-\frac{\kappa}{2}-\left(\frac{\kappa \theta}{2}-\frac{v^{2}}{8}\right) \frac{1}{\sigma_{t}^{2}}\right) \mathrm{d} s\right) .
$$

It follows from Novikov's condition that the exponent in $D_{r} \sigma_{t}^{\varepsilon}$ is negative for all choices of $\varepsilon$ and, therefore, $\left|D_{r} \sigma_{t}^{\varepsilon}\right| \leq \nu / 2$ for all $\varepsilon$. From the bounded convergence theorem we conclude that $D_{r} \sigma_{t}^{\varepsilon}$ converges to $G$ in $L^{2}(\Omega)$. Finally, Lemma 1.2 .3 of [12] implies that $\sigma_{t} \in \mathbb{D}^{1,2}$ and $D_{r} \sigma_{t}=G$.

Corollary 4.1. We have $\left|D_{r} \sigma_{t}\right| \leq(v / 2) \exp (-(\kappa / 2)(t-r))$ and $\sigma_{t} \in \mathbb{L}^{1,2}$.

Proof. Follows directly from Proposition 4.1.

Corollary 4.2. We have $v_{t} \in \mathbb{L}^{1,2}$ and, for $r<t$,

$$
D_{r} v_{t}=v \exp \left(\int_{r}^{t}\left(-\frac{\kappa}{2}-\left(\frac{\kappa \theta}{2}-\frac{v^{2}}{8}\right) \frac{1}{v_{t}}\right) \mathrm{d} s\right) \sqrt{v_{t}} .
$$

Proof. For fixed $t \in[0, T]$, we have $v_{t} \in L^{2}(\Omega)$ and

$$
v \exp \left(\int_{r}^{t}\left(-\frac{\kappa}{2}-\left(\frac{\kappa \theta}{2}-\frac{v^{2}}{8}\right) \frac{1}{v_{t}}\right) \mathrm{d} s\right) \sqrt{v_{t}} \in L^{2}(\Omega)
$$

follows again from the boundedness of the exponential. It then follows from Exercise 1.2.13 of [12] that $v_{t} \in \mathbb{D}^{1,2}$. As in Corollary 4.1, we conclude, from the explicit expression, that $v_{t} \in$ $\mathbb{L}^{1,2}$.

Let us now discuss the existence of the second Malliavin derivative of the Heston volatility. As indicated before, in order to guarantee the existence of the second Malliavin derivative we have to strengthen the conditions on the coefficients slightly. Lemma 4.2, below, will be used in the proofs of Proposition 4.2 and Proposition 5.2, below.

Lemma 4.2. Let $n \geq 2$ and $\delta:=4 \kappa \theta / v^{2}>n$, and letting $L(t)=\left(v^{2} / 4 \kappa\right)\left(1-\mathrm{e}^{-k t}\right)$ there exists a positive constant $C(n)$ such that, for all $t \in[0, T]$,

$$
\mathrm{E}\left[\frac{1}{\sigma_{t}^{n}}\right] \leq \frac{C(n)}{L(t)}\left(\frac{\mathrm{e}^{k t}}{\sigma_{0}}\right)^{n / 2-1} .
$$


Proof. From the proof of Lemma A.1 of [5] we deduce that

$$
\begin{aligned}
\mathrm{E}\left[\frac{1}{\sigma_{t}^{n}}\right]= & \frac{1}{2^{n / 2} \Gamma(n / 2) L(t)^{n / 2}} \int_{0}^{1} u^{n / 2-1}(1-u)^{2 k \theta / v^{2}-n / 2-1} \exp \left(-\frac{\sigma_{0} \mathrm{e}^{-k t} u}{2 L(t)}\right) \mathrm{d} u \\
= & \frac{1}{2^{n / 2} \Gamma(n / 2) L(t)} \int_{0}^{1} u^{n / 2-1}(1-u)^{2 k \theta / v^{2}-n / 2-1}\left(\frac{\mathrm{e}^{k t}}{\sigma_{0} u}\right)^{n / 2-1} \\
& \times\left(\frac{\sigma_{0} \mathrm{e}^{-k t} u}{L(t)}\right)^{n / 2-1} \exp \left(-\frac{\sigma_{0} \mathrm{e}^{-k t} u}{2 L(t)}\right) \mathrm{d} u .
\end{aligned}
$$

Then, using the fact that $y^{n / 2-1} \mathrm{e}^{-y} \leq C(n)$ for some positive constant $C(n)$ and any $y>0$, we can write

$$
\begin{aligned}
\mathrm{E}\left[\frac{1}{\sigma_{t}^{n}}\right] & \leq \frac{C(n)}{2^{n / 2} \Gamma(n / 2) L(t)}\left(\frac{\mathrm{e}^{k t}}{\sigma_{0}}\right)^{n / 2-1} \int_{0}^{1}(1-u)^{2 k \theta / v^{2}-n / 2-1} \mathrm{~d} u \\
& \leq \frac{C(n)}{L(t)}\left(\frac{\mathrm{e}^{k t}}{\sigma_{0}}\right)^{n / 2-1},
\end{aligned}
$$

which completes the proof.

Proposition 4.2. Assume that $4 \kappa \theta>3 v^{2}$, then $\sigma_{t} \in \mathbb{D}^{2,1}$ with

$$
\begin{aligned}
D_{\tau} D_{r} \sigma_{t}= & \frac{v^{2}}{2}\left(\frac{\kappa \theta}{2}-\frac{\nu^{2}}{8}\right) \exp \left(\int_{\tau \vee r}^{t}\left(-\frac{k}{2}-\left(\frac{k \theta}{2}-\frac{v^{2}}{8}\right) \frac{1}{\sigma_{s}^{2}}\right) \mathrm{d} s\right) \\
& \times \int_{\tau \vee r}^{t} \exp \left(\int_{\tau \vee r}^{s}\left(-\frac{k}{2}-\left(\frac{k \theta}{2}-\frac{v^{2}}{8}\right) \frac{1}{\sigma_{u}^{2}}\right) \mathrm{d} u\right) \frac{1}{\sigma_{s}^{3}} \mathrm{~d} s
\end{aligned}
$$

for $\tau<t$ and 0 otherwise. Furthermore, if $2 \kappa \theta>3 v^{2}$, we have $\sigma_{t} \in \mathbb{L}^{2,2}$ and

$$
\mathrm{E}\left|D_{\tau} D_{r} \sigma_{t}\right|^{2} \leq C\left(n, \sigma_{0}, T\right) v^{2}(t-r)(\ln t-\ln r),
$$

where $C\left(n, \sigma_{0}, T\right)$ is a constant depending on $n, \sigma_{0}$, and $T$ but not on $t, \tau$, or $\nu$.

Proof. Without loss of generality, we assume that $\tau>r$ and formally obtain

$$
\begin{aligned}
D_{\tau} D_{r} \sigma_{t}= & D_{\tau} \frac{v}{2} \exp \left(\int_{r}^{t}\left(-\frac{\kappa}{2}-\left(\frac{\kappa \theta}{2}-\frac{v^{2}}{8}\right) \frac{1}{\sigma_{t}^{2}}\right) \mathrm{d} s\right) \\
= & \frac{v}{2} \exp \left(\int_{r}^{t}\left(-\frac{\kappa}{2}-\left(\frac{\kappa \theta}{2}-\frac{v^{2}}{8}\right) \frac{1}{\sigma_{t}^{2}}\right) \mathrm{d} s\right) \int_{\tau}^{t}\left(-\left(\frac{\kappa \theta}{2}-\frac{v^{2}}{8}\right)\right)(-2) \frac{1}{\sigma_{s}^{3}} D_{\tau} \sigma_{s} \mathrm{~d} s \\
= & \frac{v^{2}}{2}\left(\frac{\kappa \theta}{2}-\frac{v^{2}}{8}\right) \exp \left(\int_{r}^{t}\left(-\frac{k}{2}-\left(\frac{k \theta}{2}-\frac{v^{2}}{8}\right) \frac{1}{\sigma_{s}^{2}}\right) \mathrm{d} s\right) \\
& \times \int_{\tau}^{t} \exp \left(\int_{\tau}^{s}\left(-\frac{k}{2}-\left(\frac{k \theta}{2}-\frac{v^{2}}{8}\right) \frac{1}{\sigma_{u}^{2}}\right) \mathrm{d} u\right) \frac{1}{\sigma_{s}^{3}} \mathrm{~d} s .
\end{aligned}
$$

Here we used the facts that $D_{\tau} \sigma_{s}=0$ for $\tau>r$ and $s \in[r, \tau)$. We will show that if $4 \kappa \theta>3 v^{2}$, this expression is contained in $L^{1}(\Omega)$. This guarantees the existence of the second Malliavin 
derivative and, furthermore, guarantees that the expression just derived is in fact the second Malliavin derivative. In order to do this, note that, for $r<\tau$ and $s<t$,

$$
\exp \left(\int_{r}^{t}\left(-\frac{\kappa}{2}-\left(\frac{\kappa \theta}{2}-\frac{v^{2}}{8}\right) \frac{1}{\sigma_{s}^{2}}\right) \mathrm{d} s\right) \leq \exp \left(\int_{\tau}^{s}\left(-\frac{\kappa}{2}-\left(\frac{\kappa \theta}{2}-\frac{v^{2}}{8}\right) \frac{1}{\sigma_{u}^{2}}\right) \mathrm{d} u\right)
$$

already follows from $2 \kappa \theta \geq v^{2}$. This implies that

$$
\begin{aligned}
\left|D_{\tau} D_{r} \sigma_{t}\right| & \leq C \int_{\tau}^{t} \exp \left(\int_{\tau}^{s}\left(-\kappa-2\left(\frac{\kappa \theta}{2}-\frac{v^{2}}{8}\right) \frac{1}{\sigma_{u}^{2}}\right) \mathrm{d} u\right) \frac{1}{\sigma_{s}^{3}} \mathrm{~d} s \\
& \leq C \int_{\tau}^{t} \exp \left(-2\left(\frac{\kappa \theta}{2}-\frac{\nu^{2}}{8}\right) \int_{\tau}^{s} \frac{1}{\sigma_{u}^{2}} \mathrm{~d} u\right) \frac{1}{\sigma_{s}^{3}} \mathrm{~d} s,
\end{aligned}
$$

where

$$
C=\frac{v^{2}}{2}\left(\frac{\kappa \theta}{2}-\frac{v^{2}}{8}\right) \leq v^{2} \frac{\kappa \theta}{4} .
$$

As in the proof of Proposition 4.1 and Corollary 4.1, it similarly follows that

$$
\left|\exp \left(-2\left(\frac{\kappa \theta}{2}-\frac{v^{2}}{8}\right) \int_{\tau}^{s} \frac{1}{\sigma_{u}^{2}} \mathrm{~d} u\right)\right| \leq 1,
$$

and, therefore, the first statement of Proposition 4.2 easily follows from Lemma 4.2 with $n=3$. The second statement can now be derived as follows. Applying the Cauchy-Schwarz inequality, we obtain

$$
\left|D_{\tau} D_{r} \sigma_{t}\right|^{2} \leq v^{2} \frac{\kappa \theta}{4}(t-\tau) \int_{\tau}^{t} \frac{1}{\sigma_{s}^{6}} \mathrm{~d} s,
$$

and, therefore, using Lemma 4.2 with $n=6$, taking into account the fact that $L(t) \geq \kappa t \mathrm{e}^{-\kappa t}$, we obtain

$$
\begin{aligned}
\mathrm{E}\left|D_{\tau} D_{r} \sigma_{t}\right|^{2} & \leq(t-r) \int_{\tau}^{t} \mathrm{E}\left[\frac{1}{\sigma_{s}^{6}}\right] \mathrm{d} s \\
& \leq C(n) \frac{\theta v^{2}}{4 \sigma_{0}^{2}}(t-r) \int_{\tau}^{t} \frac{\mathrm{e}^{k s}}{s}\left(\mathrm{e}^{k s}\right)^{2} \mathrm{~d} s \\
& \leq C\left(n, \sigma_{0}, T\right) v^{2}(t-r)(\ln t-\ln r),
\end{aligned}
$$

where $C\left(n, \sigma_{0}, T\right)=C(n)\left(\theta / 4 \sigma_{0}^{2}\right) \mathrm{e}^{\kappa T}$.

\section{An approximate option pricing formula for the Heston model}

Let us consider the Heston stochastic volatility model with correlation $\rho$, which consists of a stock, a money market account with deterministic interest rate $r$, and the volatility process $v_{t}$ satisfying (1.1) and (1.2), where we assume that $\mathrm{d} B_{t} \mathrm{~d} W_{t}=\rho \mathrm{d} t$ with $\rho \in(-1,1)$. It is well known that there exists a two-dimensional Brownian motion $\left(Z_{t}, W_{t}\right)^{\top}$ on a filtered probability space $\left(\Omega,\left(\mathcal{F}_{t}\right), \mathrm{P}\right)$ satisfying the usual conditions that $B_{t}=\rho W_{t}+\sqrt{1-\rho^{2}} Z_{t}$. It is helpful in the following to think of the dynamic described by (1.1) and (1.2) as driven by $\left(Z_{t}, W_{t}\right)^{\top}$ rather than by $\left(B_{t}, W_{t}\right)^{\top}$. We also assume that the dynamic is satisfied under the risk neutral measure chosen by the market and that this risk neutral measure is given by $\mathrm{P}$. This implies that $b=r$. In the following we work with the logarithmic price $X_{t}=\ln \left(S_{t}\right)$ rather than with 
the actual price. The price of a contingent claim $h\left(X_{T}\right)$ at time $t$ can then be computed via the formula $V_{t}=\mathrm{e}^{-r(T-t)} \mathrm{E}\left[h\left(X_{T}\right) \mid \mathcal{F}_{t}\right]$. In the following we will consider the call payoff function $h(x)=\left(\mathrm{e}^{x}-K\right)_{+}$for some fixed $K$, and denote with $\operatorname{BS}(t, x, \sigma)$ the price at time $t$ of the corresponding contingent claim in the standard Black-Scholes model with constant volatility $\sigma$, given that the $\log$ price at time $t$ is $x$. Furthermore, we denote by

$$
\vartheta_{t}:=\sqrt{\frac{1}{T-t} \int_{t}^{T} \sigma_{s}^{2} \mathrm{~d} s}
$$

the average Heston future volatility starting from time $t$, and denote by $D$ the Malliavin derivative operator with respect to the Brownian motion $W$. Proposition 5.1, below, is in line with Theorem 3 of [1] and Theorem 3 of [3].

Proposition 5.1. Consider the Heston model, and assume that $2 \kappa \theta \geq v^{2}$. Then

$$
V_{t}=\mathrm{E}\left[\mathrm{BS}\left(t, X_{t}, \vartheta_{t}\right) \mid \mathcal{F}_{t}\right]+\frac{\rho}{2} \mathrm{E}\left[\int_{t}^{T} \mathrm{e}^{-r(s-t)} H\left(s, X_{s}, \vartheta_{s}\right) \Lambda_{s} \mathrm{~d} s \mid \mathcal{F}_{t}\right],
$$

where

$$
H(s, x, \sigma):=\left(\frac{\partial^{3}}{\partial x^{3}}-\frac{\partial^{2}}{\partial x^{2}}\right) \mathrm{BS}(s, x, \sigma) \quad \text { and } \quad \Lambda_{s}:=\left(\int_{s}^{T} D_{s} \sigma_{r}^{2} \mathrm{~d} r\right) \sigma_{s} .
$$

Proof. Follows from Proposition 4.1 in connection with Theorem 3 of [3].

It follows from the classical Hull and White formula (see [9]) that $\mathrm{E}\left[\mathrm{BS}\left(t, X_{t}, \vartheta_{t}\right) \mid \mathcal{F}_{t}\right]$ is the price of the contingent claim in the Heston model without correlation. Proposition 5.1 therefore extends the classical Hull and White formula to the Heston model with correlation, and gives interesting insight into how the correlation effects option prices. It states that this correlation effect is explicitly given by the second summand in (5.1). This fact is very useful in order to study price sensitivities with respect to $\rho$ in the Heston stochastic volatility model or for the purpose of calibration of the model. In the following we propose various approximations for the correlation effect, which are computationally more accessible, and derive bounds for the error of these approximations. For this, we consider maturities $T-t<1$ and assume that $\sigma_{0}^{2}<1$. From a financial point of view, both assumptions are reasonable, as market parameters are all denoted on a yearly scale and maturity times of options are mostly less than one year, while annual volatility is usually in the range of less than $10 \%$.

Lemma 5.1. Assume that $2 \kappa \theta \geq v^{2}$, then

$$
\mathrm{E}\left[\left(\int_{t}^{T} \sigma_{s}^{2} \mathrm{~d} s\right)^{-1 / 2} \mid \mathcal{F}_{t}\right] \leq \frac{C\left(\sigma_{t}\right)}{(T-t)},
$$

where $C\left(\sigma_{t}\right)$ is a constant depending on the current level of volatility but not on t explicitly.

Proof. Since we are in a Markovian framework, without loss of generality, we can assume that $t=0$ and we can replace all the conditional expectations by their unconditional counterparts. Using the identity

$$
\frac{1}{x^{\alpha}}=\frac{1}{\Gamma(\alpha)} \int_{0}^{\infty} u^{\alpha-1} \mathrm{e}^{-u x} \mathrm{~d} u
$$


while choosing $x=\int_{0}^{T} \sigma_{s}^{2} \mathrm{~d} s$ and $\alpha=\frac{1}{2}$, we conclude that

$$
\mathrm{E}\left[\left(\int_{0}^{T} \sigma_{s}^{2} \mathrm{~d} s\right)^{-1 / 2}\right]=\frac{1}{\Gamma(1 / 2)} \int_{0}^{\infty} u^{-1 / 2} \mathrm{E}\left[\exp \left(-u\left(\int_{0}^{T} \sigma_{s}^{2} \mathrm{~d} s\right)\right)\right] \mathrm{d} u .
$$

Since the Heston volatility is in fact a time transformed and scaled squared Bessel process, we can write

$$
\begin{aligned}
\mathrm{E}\left[\exp \left(-u\left(\int_{0}^{T} \sigma_{s}^{2} \mathrm{~d} s\right)\right)\right] & \leq \mathrm{E}\left[\exp \left(-u\left(\int_{0}^{T} \mathrm{e}^{-\kappa s} \eta\left(\frac{v^{2}}{4 \kappa}\left(\mathrm{e}^{\kappa s}-1\right)\right) \mathrm{d} s\right)\right)\right] \\
& \leq \mathrm{E}\left[\exp \left(-u\left(\frac{4}{v^{2}} \int_{0}^{\left(v^{2} / 4 \kappa\right)\left(\mathrm{e}^{\kappa T}-1\right)} \eta(m) \mathrm{d} m\right)\right)\right],
\end{aligned}
$$

where $\eta$ is a squared Bessel process of dimension $\delta=4 \kappa \theta / \nu^{2}$.

It follows from the scaling property of Bessel processes, similarly as in [4, p. 377] or Proposition 5.5.3 of [10], that, for every constant $b$ and for every $t>0$,

$$
\mathrm{E}\left[\exp \left(-\frac{b^{2}}{2}\left(\int_{0}^{t} \eta(\alpha) \mathrm{d} \alpha\right)\right)\right]=(\cosh (b t))^{-\delta / 2} \exp \left(-\frac{b x}{2} \tanh (b t)\right) .
$$

By substitution, it follows that

$$
\begin{aligned}
& \mathrm{E}\left[\exp \left(-u\left(\frac{4}{v^{2}} \int_{0}^{\left(v^{2} / 4 \kappa\right)\left(\mathrm{e}^{\kappa T}-1\right)} \eta(\alpha) \mathrm{d} \alpha\right)\right)\right] \\
& \quad \leq\left(\cosh \left(\frac{v}{2 \kappa}\left(\mathrm{e}^{\kappa T}-1\right) \sqrt{2 u}\right)\right)^{-\delta / 2} \exp \left(-\frac{\sqrt{2 u}}{v} \sigma_{0}^{2} \tanh \left(\frac{v}{2 \kappa}\left(\mathrm{e}^{\kappa T}-1\right) \sqrt{2 u}\right)\right) .
\end{aligned}
$$

Using the latter together with (5.2), we conclude that

$$
\begin{aligned}
\mathrm{E}\left[\left(\int_{0}^{T} \sigma_{s}^{2} \mathrm{~d} s\right)^{-1 / 2}\right] \leq \frac{1}{\Gamma(1 / 2)} \int_{0}^{\infty} & u^{-1 / 2}\left(\cosh \left(\frac{v}{2 \kappa}\left(\mathrm{e}^{\kappa T}-1\right) \sqrt{2 u}\right)\right)^{-\delta / 2} \\
& \times \exp \left(-\frac{\sqrt{2 u}}{v} \sigma_{0}^{2} \tanh \left(\frac{v}{2 \kappa}\left(\mathrm{e}^{\kappa T}-1\right) \sqrt{2 u}\right)\right) \mathrm{d} u .
\end{aligned}
$$

Now by substitution of $\tilde{m}:=(v / 2 \kappa)\left(\mathrm{e}^{\kappa T}-1\right) \sqrt{2 u}$ we obtain

$$
\mathrm{E}\left[\left(\int_{0}^{T} \sigma_{s}^{2} \mathrm{~d} s\right)^{-1 / 2}\right] \leq \frac{C_{1}}{\mathrm{e}^{\kappa T}-1} \int_{0}^{\infty}(\cosh (\tilde{m}))^{-\delta / 2} \exp \left(-\frac{\tilde{m} \sqrt{2} \kappa}{v^{2}\left(\mathrm{e}^{\kappa T}-1\right)} \sigma_{0}^{2} \tanh (\tilde{m})\right) \mathrm{d} \tilde{m},
$$

where $C_{1}:=2 \sqrt{2} \kappa / \nu$ is a constant. It is not difficult to see that, since $\delta \geq 2$, the integral on the right-hand side is finite and the last inequality can be written as

$$
\mathrm{E}\left[\left(\int_{0}^{T} \sigma_{s}^{2} \mathrm{~d} s\right)^{-1 / 2}\right] \leq \frac{C_{1} I\left(\sigma_{0}\right)}{\mathrm{e}^{\kappa T}-1}
$$

where $I\left(\sigma_{0}\right)$ denotes the value of the integral. Now we can use the fact that, for positive $\kappa$, we have $\left(\mathrm{e}^{\kappa T}-1\right) \geq \kappa T$ and obtain

$$
\mathrm{E}\left[\left(\int_{0}^{T} \sigma_{s}^{2} \mathrm{~d} s\right)^{-1 / 2}\right] \leq \frac{C_{1} I\left(\sigma_{0}\right)}{\mathrm{e}^{\kappa T}-1} \leq \frac{C_{1} I\left(\sigma_{0}\right)}{\kappa T}=\frac{C\left(\sigma_{0}\right)}{T},
$$

where $C\left(\sigma_{0}\right)=C_{1} I\left(\sigma_{0}\right) / \kappa$. 
Proposition 5.2. Consider the Heston model, and assume that $2 \kappa \theta \geq 3 v^{2}$. For $t \in[0, T]$, there exists a constant $C\left(\sigma_{t}\right)$ which does not depend on $t, v$, and $\rho$ explicitly, such that

$$
\left|V_{t}-\mathrm{E}\left[\mathrm{BS}\left(t, X_{t} ; \vartheta_{t}\right)+\frac{\rho}{2} H\left(t, X_{t}, \vartheta_{t}\right)\left(\int_{t}^{T} \Lambda_{s} \mathrm{~d} s\right) \mid \mathscr{F}_{t}\right]\right| \leq C\left(\sigma_{t}\right) v^{2} \rho^{2}(T-t)
$$

Proof. From Proposition 5.1, it follows that

$$
\begin{aligned}
\mid V_{t}- & \mathrm{E}\left[\mathrm{BS}\left(t, X_{t}, \vartheta_{t}\right)+\frac{\rho}{2} H\left(t, X_{t}, \vartheta_{t}\right)\left(\int_{t}^{T} \Lambda_{s} \mathrm{~d} s\right) \mid \mathcal{F}_{t}\right] \mid \\
& =\left|\mathrm{E}\left[\frac{\rho}{2} \int_{t}^{T} \mathrm{e}^{-r(s-t)} H\left(s, X_{s}, \vartheta_{s}\right) \Lambda_{s} \mathrm{~d} s-\frac{\rho}{2} H\left(t, X_{t}, \vartheta_{t}\right)\left(\int_{t}^{T} \Lambda_{s} \mathrm{~d} s\right) \mid \mathcal{F}_{t}\right]\right| .
\end{aligned}
$$

Let us now consider the process $(\rho / 2) \mathrm{e}^{-r t} H\left(t, X_{t}, \vartheta_{t}\right)\left(\int_{t}^{T} \Lambda_{u} \mathrm{~d} u\right)$. Obviously, this process vanishes at $t=T$ and it follows from Proposition 3.1, as in the proof of Proposition 7 of [1], that

$$
\begin{aligned}
\mathrm{E}\left[\frac{\rho}{2} \int_{t}^{T} \mathrm{e}^{-r(s-t)} H\left(s, X_{s}, \vartheta_{s}\right) \Lambda_{s} \mathrm{~d} s-\frac{\rho}{2} H\left(t, X_{t}, \vartheta_{t}\right)\left(\int_{t}^{T} \Lambda_{u} \mathrm{~d} u\right) \mid \mathcal{F}_{t}\right] \\
=\mathrm{E}\left[\frac{\rho^{2}}{8} \int_{t}^{T} \mathrm{e}^{-r(s-t)} G\left(s, X_{s}, \vartheta_{s}\right)\left(\int_{s}^{T} \Lambda_{r} \mathrm{~d} r\right) \Lambda_{s} \mathrm{~d} s\right. \\
\left.\quad+\frac{\rho^{2}}{4} \int_{t}^{T} \mathrm{e}^{-r(s-t)} \frac{\partial H}{\partial x}\left(s, X_{s}, \vartheta_{s}\right)\left(\int_{s}^{T} D_{s} \Lambda_{r} \mathrm{~d} r\right) \sigma_{s} \mathrm{~d} s \mid \mathcal{F}_{t}\right] \\
=: A_{1}+A_{2}
\end{aligned}
$$

where

$$
G\left(s, X_{s}, \vartheta_{s}\right)=\left(\frac{\partial^{3}}{\partial x^{3}}-\frac{\partial^{2}}{\partial x^{2}}\right) H\left(s, X_{s}, \vartheta_{s}\right)
$$

and $A_{1}$ and $A_{2}$ respectively correspond to the first and second summands above. Let $g_{t}$ denote the $\sigma$-algebra generated by the Brownian motion, $W_{t}$, which drives the Heston volatility. Now the proof will be decomposed into two steps.

Step 1. Let us study the $A_{1}$ term. From Lemma 2 of [3] we conclude that, for $0 \leq t \leq s \leq T$, we have

$$
\left|\mathrm{E}\left[\frac{\partial^{n}}{\partial x^{n}}\left(\frac{\partial^{2}}{\partial x^{2}}-\frac{\partial}{\partial x}\right) \mathrm{BS}\left(s, X_{s}, \vartheta_{s}\right) \mid g_{t}\right]\right| \leq C\left(\sigma_{t}\right) \rho\left(\int_{t}^{T} \sigma_{s}^{2} \mathrm{~d} s\right)^{-(n+1) / 2} .
$$

Here $C\left(\sigma_{t}\right)$ is a constant whose value depends on the current value of $\sigma_{t}$. Hölder's inequality and the fact that $D_{r} \sigma_{\theta}^{2}=2 \sigma_{\theta} D_{r} \sigma_{\theta}$ allow us to write

$$
\int_{t}^{T}\left(\int_{s}^{T} \Lambda_{r} \mathrm{~d} r\right) \Lambda_{s} \mathrm{~d} s \leq\left(\int_{t}^{T} \sigma_{s}^{2} \mathrm{~d} s\right)^{2}\left(\int_{t}^{T}\left(\int_{r}^{T}\left(D_{r} \sigma_{\theta}\right)^{2} \mathrm{~d} \theta\right) \mathrm{d} r\right)
$$


Then, (5.3) and (5.4) yield

$$
\begin{aligned}
A_{1} \leq C \frac{\rho^{2}}{8} \mathrm{E}[ & \left(\left(\int_{t}^{T} \sigma_{s}^{2} \mathrm{~d} s\right)^{-5 / 2}+\left(\int_{t}^{T} \sigma_{s}^{2} \mathrm{~d} s\right)^{-2}\right. \\
& \left.\left.+\left(\int_{t}^{T} \sigma_{s}^{2} \mathrm{~d} s\right)^{-3 / 2}\right) \int_{t}^{T}\left(\int_{s}^{T} \Lambda_{r} \mathrm{~d} r\right) \Lambda_{s} \mathrm{~d} s \mid \mathcal{F}_{t}\right] \\
\leq C \frac{\rho^{2}}{8} \mathrm{E}[ & \left(1+\left(\int_{t}^{T} \sigma_{s}^{2} \mathrm{~d} s\right)^{-1 / 2}+\left(\int_{t}^{T} \sigma_{s}^{2} \mathrm{~d} s\right)^{1 / 2}\right) \\
& \left.\times\left(\int_{t}^{T}\left(\int_{r}^{T}\left(D_{r} \sigma_{\theta}\right)^{2} \mathrm{~d} \theta\right) \mathrm{d} r\right) \mid \mathcal{F}_{t}\right],
\end{aligned}
$$

and now, using the fact that $\left(D_{r} \sigma_{\theta}\right)^{2}$ is bounded by $v^{2}$, it follows that

$$
A_{1} \leq C v^{2} \rho^{2}(T-t)^{2} \mathrm{E}\left[\left(1+\left(\int_{t}^{T} \sigma_{s}^{2} \mathrm{~d} s\right)^{-1 / 2}+\left(\int_{t}^{T} \sigma_{s}^{2} \mathrm{~d} s\right)^{1 / 2}\right) \mid \mathcal{F}_{t}\right] .
$$

Lemma 5.1 and the fact that $\mathrm{E}\left[\int_{t}^{T} \sigma_{s}^{2} \mathrm{~d} s\right]^{1 / 2}$ is finite, as well as assuming that $T-t<1$, now imply that $A_{1} \leq C\left(\sigma_{t}\right) v^{2} \rho^{2}(T-t)$.

Step 2. Let us study the $A_{2}$ term. Again using Hölder's inequality, we can write

$$
\begin{aligned}
\int_{t}^{T}\left(\int_{s}^{T} D_{s} \Lambda_{r} \mathrm{~d} r\right) \sigma_{s} \mathrm{~d} s \leq & \left(\int_{t}^{T} \sigma_{s}^{2} \mathrm{~d} s\right) \int_{t}^{T} \int_{r}^{T}\left(D_{r} \sigma_{\alpha}\right)^{2} \mathrm{~d} \alpha \mathrm{d} r \\
& +\left(\int_{t}^{T} \sigma_{s}^{2} \mathrm{~d} s\right)^{3 / 2}\left(\int_{t}^{T}\left(\int_{s}^{T}\left(\int_{r}^{T}\left(D_{s} D_{r} \sigma_{\alpha}\right)^{2} \mathrm{~d} \alpha\right) \mathrm{d} r\right) \mathrm{d} s\right)^{1 / 2} .
\end{aligned}
$$

Then, using (5.3) and (5.5) in a similar way as in step 1, we obtain

$$
\begin{aligned}
A_{2} \leq & \frac{\rho^{2}}{4} \mathrm{E}\left[\left(1+\left(\int_{t}^{T} \sigma_{s}^{2} \mathrm{~d} s\right)^{-1 / 2}\right)\left(\int_{t}^{T} \int_{r}^{T}\left(D_{r} \sigma_{\alpha}\right)^{2} \mathrm{~d} \alpha \mathrm{d} r\right) \mid \mathcal{F}_{t}\right] \\
& +C\left(\sigma_{t}\right) \frac{\rho^{2}}{4} \mathrm{E}\left[\int_{t}^{T}\left(\int_{s}^{T}\left(\int_{r}^{T}\left(D_{s} D_{r} \sigma_{\alpha}\right)^{2} \mathrm{~d} \alpha\right) \mathrm{d} r\right) \mathrm{d} s \mid \mathcal{F}_{t}\right] .
\end{aligned}
$$

Now Proposition 4.1, Proposition 4.2, and our assumption that $T-t<1$ enable us to deduce that $A_{2} \leq C\left(\sigma_{t}\right) v^{2} \rho^{2}(T-t)$.

Remark 5.1. Let us briefly illustrate how the result in Proposition 5.2 should be interpreted in a dynamic framework. As one can obviously see, the approximation is getting better with a quadratic rate, as the factor $v$ decreases. The situation is similar for $\rho$. As the constant $C\left(\sigma_{t}\right)$, however, depends implicitly on $t$ through $\sigma_{t}$, we cannot say that, as time to maturity decreases, our approximation is getting better in general. In fact, a large change in the volatility during a trading day may lead to the result that our approximation tomorrow is in fact worse than today. This effect, however, is entirely caused by the random volatility. Putting aside this effect and fixing the volatility artificially in time, the accuracy of the approximation then increases at least linearly with decreasing time to maturity. 
Let us now consider the following approximation for the correlation effect:

$$
\frac{\rho}{2} H\left(t, X_{t}, \vartheta_{t}^{*}\right) \mathrm{E}\left[\int_{t}^{T} \Lambda_{s} \mathrm{~d} s \mid \mathcal{F}_{t}\right]
$$

where

$$
\vartheta_{t}^{*}=\sqrt{\frac{1}{T-t} \int_{t}^{T} \mathrm{E}\left[\sigma_{s}^{2} \mid \mathcal{F}_{t}\right] \mathrm{d} s}
$$

Let us consider the following as an approximation of the option price:

$$
\mathrm{BS}\left(t, X_{t} ; \vartheta_{t}^{*}\right)+\frac{\rho}{2} H\left(t, X_{t}, \vartheta_{t}^{*}\right) \mathrm{E}\left[\left(\int_{t}^{T} \Lambda_{s} \mathrm{~d} s\right) \mid \mathcal{F}_{t}\right] .
$$

Later, we will need Lemma 5.2, below, which is related to (5.3) but, for the specific case considered here, gives a slightly better approximation.

Lemma 5.2. Let $\mathrm{BS}(t, x, \sigma)$ denote the Black-Scholes price in the log-stock price $x$. Then there exists a constant such that, for all times to maturity $T-t<1$, we have

$$
\left|\left(\frac{\partial^{2}}{\partial x^{2}}-\frac{\partial}{\partial x}\right)^{2} \operatorname{BS}(t, x, \sigma)\right| \leq C \sigma^{-2}(T-t)^{-3 / 2} .
$$

Proof. Applying the chain rule of differential calculus with $S=\mathrm{e}^{x}$, we obtain

$$
\begin{gathered}
\frac{\partial}{\partial x} \mathrm{BS}(t, x, \sigma)=N\left(d_{1}\right) \mathrm{e}^{x}, \\
\frac{\partial^{2}}{\partial x^{2}} \mathrm{BS}(t, x, \sigma)=\left(\frac{\partial}{\partial x}\left(N\left(d_{1}\right)\right)+N\left(d_{1}\right)\right) \mathrm{e}^{x}=\left(\frac{N^{\prime}\left(d_{1}\right)}{\sigma \sqrt{T-t}}+N\left(d_{1}\right)\right) \mathrm{e}^{x},
\end{gathered}
$$

where $d_{1}$ denotes the classical Black-Scholes parameter

$$
d_{1}=\frac{x-\ln K+\left(r+\sigma^{2} / 2\right)(T-t)}{\sigma \sqrt{T-t}}
$$

and $N(\cdot)$ is the standard normal distribution function. Therefore,

$$
\left(\frac{\partial^{2}}{\partial x^{2}}-\frac{\partial}{\partial x}\right) \operatorname{BS}(t, x, \sigma)=\frac{N^{\prime}\left(d_{1}\right)}{\sigma \sqrt{T-t}} \mathrm{e}^{x} .
$$

Furthermore, differentiation now shows that

$$
\begin{aligned}
\left(\frac{\partial^{2}}{\partial x^{2}}-\frac{\partial}{\partial x}\right)^{2} \operatorname{BS}(t, x, \sigma) & =\left(\frac{\partial^{2}}{\partial x^{2}}-\frac{\partial}{\partial x}\right)\left(\frac{N^{\prime}\left(d_{1}\right)}{\sigma \sqrt{T-t}} \mathrm{e}^{x}\right) \\
& =\left(\frac{N^{\prime \prime \prime}\left(d_{1}\right)}{\sigma^{2}(T-t)^{3 / 2}}+\frac{N^{\prime \prime}\left(d_{1}\right)}{\sigma(T-t)}\right) \mathrm{e}^{x}
\end{aligned}
$$

The result then follows, since all the derivatives of the normal distribution function $N(x)$ are bounded and, furthermore, $(T-t)^{3 / 2}$ dominates $(T-t)$ for $T-t<1$.

Proposition 5.3, below, represents an analytic result on the quality of approximation (5.7). 
Proposition 5.3. Assume that $2 \kappa \theta \geq 3 v^{2}$, and define $\vartheta_{t}^{*}$ as before for $t \in[0, T]$. Then there exists a constant $C\left(\sigma_{t}\right)$ which does not depend explicitly on $t$ and $v$ such that

$$
\left|V_{t}-\mathrm{BS}\left(t, X_{t} ; \vartheta_{t}^{*}\right)-\frac{\rho}{2} H\left(t, X_{t}, \vartheta_{t}^{*}\right) \mathrm{E}\left[\left(\int_{t}^{T} \Lambda_{s} \mathrm{~d} s\right) \mid \mathcal{F}_{t}\right]\right| \leq C\left(\sigma_{t}\right) v^{2}(T-t) .
$$

Proof. We can write

$$
\begin{aligned}
\mid V_{t}- & \mathrm{BS}\left(t, X_{t} ; \vartheta_{t}^{*}\right)-\frac{\rho}{2} H\left(t, X_{t}, \vartheta_{t}^{*}\right) \mathrm{E}\left[\left(\int_{t}^{T} \Lambda_{s} \mathrm{~d} s\right) \mid \mathcal{F}_{t}\right] \mid \\
\leq & \left|V_{t}-\mathrm{E}\left[\mathrm{BS}\left(t, X_{t} ; \vartheta_{t}\right)+\frac{\rho}{2} H\left(t, X_{t}, \vartheta_{t}\right)\left(\int_{t}^{T} \Lambda_{s} \mathrm{~d} s\right) \mid \mathcal{F}_{t}\right]\right| \\
& +\left|\mathrm{E}\left[\mathrm{BS}\left(t, X_{t} ; \vartheta_{t}\right) \mid \mathcal{F}_{t}\right]-\mathrm{BS}\left(t, X_{t} ; \vartheta_{t}^{*}\right)\right| \\
& +\frac{\rho}{2}\left|\mathrm{E}\left[\left(H\left(t, X_{t}, \vartheta_{t}\right)-H\left(t, X_{t}, \vartheta_{t}^{*}\right)\right)\left(\int_{t}^{T} \Lambda_{s} \mathrm{~d} s\right) \mid \mathcal{F}_{t}\right]\right| \\
= & B_{1}+B_{2}+B_{3},
\end{aligned}
$$

where $B_{1}, B_{2}$, and $B_{3}$ respectively correspond to the first, second, and third summands above. We conclude from Proposition 5.2 that $B_{1} \leq C\left(\sigma_{t}\right) v^{2} \rho^{2}(T-t)$, and we are left with expressions $B_{2}$ and $B_{3}$. Let us study expression $B_{2}$ first. Note that

$$
\vartheta_{t}^{*}=\sqrt{\frac{1}{T-t}\left(M_{t}-\int_{0}^{t} \sigma_{s}^{2} \mathrm{~d} s\right)}, \quad \vartheta_{t}=\sqrt{\frac{1}{T-t}\left(M_{T}-\int_{0}^{t} \sigma_{s}^{2} \mathrm{~d} s\right)},
$$

where $M_{t}:=\int_{0}^{T} \mathrm{E}\left[\sigma_{s}^{2} \mid \mathcal{F}_{t}\right] \mathrm{d} s$. It is not difficult to verify the following:

$$
\begin{gathered}
M_{t}=\int_{t}^{T}\left(\sigma_{t}^{2} \mathrm{e}^{-\kappa(s-t)}+\theta\left(1-\mathrm{e}^{-\kappa(s-t)}\right)\right) \mathrm{d} s+\int_{0}^{t} \sigma_{s}^{2} \mathrm{~d} s \\
\mathrm{~d} M_{t}=\int_{t}^{T}\left(\kappa \sigma_{t}^{2} \mathrm{e}^{-\kappa(s-t)} \mathrm{d} t+\mathrm{e}^{-\kappa(s-t)} \mathrm{d} \sigma_{t}^{2}-\kappa \theta \mathrm{e}^{-\kappa(s-t)}\right) \mathrm{d} s=\nu \sigma_{t}\left(\int_{t}^{T} \mathrm{e}^{-\kappa(s-t)} \mathrm{d} s\right) \mathrm{d} W_{t} .
\end{gathered}
$$

Using the classical Itô formula and the relationship between the Greeks,

$$
\frac{\partial \mathrm{BS}}{\partial \sigma}(s, x, \sigma) \frac{1}{\sigma(T-s)}=\left(\frac{\partial^{2}}{\partial x^{2}}-\frac{\partial}{\partial x}\right) \mathrm{BS}(s, x, \sigma),
$$

we deduce that

$$
\begin{aligned}
B_{2}= & \mathrm{E}\left[\mathrm{BS}\left(t, X_{t} ; \vartheta_{t}\right) \mid \mathcal{F}_{t}\right]-\mathrm{BS}\left(t, X_{t} ; \vartheta_{t}^{*}\right) \\
= & \mathrm{E}\left[\mathrm{BS}\left(t, X_{t} ; \sqrt{\frac{1}{T-t}\left(M_{T}-\int_{0}^{t} \sigma_{s}^{2} \mathrm{~d} s\right)}\right) \mid \mathcal{F}_{t}\right] \\
& -\mathrm{E}\left[\mathrm{BS}\left(t, X_{t} ; \sqrt{\frac{1}{T-t}\left(M_{t}-\int_{0}^{t} \sigma_{s}^{2} \mathrm{~d} s\right)} \mid \mathcal{F}_{t}\right]\right. \\
= & \frac{v^{2}}{8} \mathrm{E}\left[\int_{t}^{T}\left(\frac{\partial^{2}}{\partial x^{2}}-\frac{\partial}{\partial x}\right)^{2} \mathrm{BS}\left(t, X_{t} ; \sqrt{\frac{1}{T-t}\left(M_{u}-\int_{0}^{t} \sigma_{s}^{2} \mathrm{~d} s\right)}\right)\right. \\
& \left.\times\left(\int_{u}^{T} \mathrm{e}^{-k(s-u)} \mathrm{d} s\right)^{2} \sigma_{u}^{2} \mathrm{~d} u \mid \mathcal{F}_{t}\right] .
\end{aligned}
$$


We can now conclude from Lemma 5.2 that there exists a constant $C>0$ such that

$$
\begin{aligned}
B_{2} & \leq v^{2} \mathrm{E}\left[\int_{t}^{T} C(T-t)^{-3 / 2}\left(\frac{1}{T-t}\left(M_{u}-\int_{0}^{t} \sigma_{s}^{2} \mathrm{~d} s\right)\right)^{-1}\left(\int_{u}^{T} \mathrm{e}^{-\kappa(s-u)} \mathrm{d} s\right)^{2} \sigma_{u}^{2} \mathrm{~d} u \mid \mathcal{F}_{t}\right] \\
& =C v^{2}(T-t)^{-1 / 2} \mathrm{E}\left[\int_{t}^{T}\left(\int_{t}^{T} \mathrm{E}\left[\sigma_{s}^{2} \mid \mathcal{F}_{u}\right] \mathrm{d} s\right)^{-1}\left(\int_{u}^{T} \mathrm{e}^{-\kappa(s-u)} \mathrm{d} s\right)^{2} \sigma_{u}^{2} \mathrm{~d} u \mid \mathcal{F}_{t}\right] .
\end{aligned}
$$

Now, using the fact that $t<u$, the definition of $M_{t}$, and (5.8), we obtain

$$
\begin{aligned}
\left(\int_{t}^{T} \mathrm{E}\left[\sigma_{s}^{2} \mid \mathcal{F}_{u}\right] \mathrm{d} s\right)^{-1} & \leq\left(\int_{u}^{T} \mathrm{E}\left[\sigma_{s}^{2} \mid \mathcal{F}_{u}\right] \mathrm{d} s\right)^{-1} \\
& =\left(\int_{u}^{T}\left(\sigma_{u}^{2} \mathrm{e}^{-\kappa(s-u)}+\theta\left(1-\mathrm{e}^{-\kappa(s-u)}\right)\right) \mathrm{d} s\right)^{-1} \\
& \leq \sigma_{u}^{-2}\left(\int_{u}^{T} \mathrm{e}^{-\kappa(s-u)} \mathrm{d} s\right)^{-1}
\end{aligned}
$$

as $\theta\left(1-\mathrm{e}^{-\kappa(s-u)}\right) \geq 0$ for all $s \geq u$. Back substitution gives

$$
\begin{aligned}
B_{2} & \leq C v^{2}(T-t)^{-1 / 2} \mathrm{E}\left[\int_{t}^{T} \sigma_{u}^{-2}\left(\int_{u}^{T} \mathrm{e}^{-\kappa(s-u)} \mathrm{d} s\right)^{-1}\left(\int_{u}^{T} \mathrm{e}^{-\kappa(s-u)} \mathrm{d} s\right)^{2} \sigma_{u}^{2} \mathrm{~d} u \mid \mathcal{F}_{t}\right] \\
& \leq C \nu^{2}(T-t)^{-1 / 2} \int_{t}^{T} \int_{u}^{T} \mathrm{e}^{-\kappa(s-u)} \mathrm{d} s \mathrm{~d} u \\
& \leq \frac{C}{\kappa} v^{2}(T-t)^{3 / 2},
\end{aligned}
$$

where we used the facts that $t \leq u \leq s$ and $0<\mathrm{e}^{-\kappa(s-u)} \leq 1$. The last expression above is bounded by $(C / \kappa) v^{2}(T-t)$ for all $T-t<1$. Let us finally consider expression $B_{3}$. Proposition 3.1 and (5.9) imply that

$$
\begin{aligned}
B_{3}= & \mathrm{E}\left[\left(H\left(t, X_{t}, \vartheta_{t}\right)-H\left(t, X_{t}, \vartheta_{t}^{*}\right)\right)\left(\int_{t}^{T} \Lambda_{s} \mathrm{~d} s\right) \mid \mathcal{F}_{t}\right] \\
= & \frac{v}{2} \mathrm{E}\left[\int_{t}^{T}\left(\frac{\partial^{2}}{\partial x^{2}}-\frac{\partial}{\partial x}\right) H\left(t, X_{t}, \sqrt{\frac{1}{T-t}\left(M_{u}-\int_{0}^{t} \sigma_{s}^{2} \mathrm{~d} s\right)}\right)\right. \\
& \left.\times\left(D_{u} \int_{u}^{T} \Lambda_{s} \mathrm{~d} s\right) \sigma_{u}\left(\int_{u}^{T} \mathrm{e}^{-\kappa(s-u)} \mathrm{d} s\right) \mathrm{d} u \mid \mathcal{F}_{t}\right] \\
+ & \frac{v^{2}}{8} \mathrm{E}\left[\int_{t}^{T}\left(\frac{\partial^{2}}{\partial x^{2}}-\frac{\partial}{\partial x}\right)^{2} H\left(t, X_{t}, \sqrt{\frac{1}{T-t}\left(M_{u}-\int_{0}^{t} \sigma_{s}^{2} \mathrm{~d} s\right)}\right)\right. \\
+ & \times\left(\int_{t}^{T} H\left(t, X_{t}, \sqrt{\frac{1}{T-t}\left(M_{u}-\int_{0}^{t} \sigma_{s}^{2} \mathrm{~d} s\right)}\right) \sigma_{u}^{2} \mathrm{~d} u .\right.
\end{aligned}
$$

Now, arguments similar to those used for $B_{2}$ give us $B_{3} \leq C\left(\sigma_{t}\right) v^{2}(T-t)$. 
Let us now make things more transparent by evaluating the expression

$$
\frac{1}{2} H\left(t, X_{t}, \vartheta_{t}^{*}\right) \mathrm{E}\left[\int_{t}^{T} \Lambda_{s} \mathrm{~d} s \mid \mathcal{F}_{t}\right]
$$

which determines the effect of correlation on option prices in the Heston model. We have to evaluate

$$
\vartheta_{t}^{*} \quad \text { and } \quad \mathrm{E}\left[\int_{t}^{T} \Lambda_{s} \mathrm{~d} s \mid \mathcal{F}_{t}\right] .
$$

Since the framework is a Markovian one, we can assume, without loss of generality, that $t=0$. In this case we have to evaluate the quantities

$$
\vartheta_{0}^{*}=\sqrt{\left(\frac{1}{T}\right) \int_{0}^{T} \mathrm{E}\left[\sigma_{s}^{2}\right] \mathrm{d} s} \text { and } \mathrm{E}\left[\int_{0}^{T} \Lambda_{s} \mathrm{~d} s\right] .
$$

Let us start with the computation of $\vartheta_{t}^{*}$. It follows, from $\sigma_{s}=\sqrt{v_{s}}$ and the dynamics of $v_{s}$ by taking expectations and solving the corresponding ordinary differential equation for the expectation, that

$$
\mathrm{E}\left[\sigma_{s}^{2}\right]=\mathrm{E}\left[v_{s}\right]=\theta+\left(v_{0}-\theta\right) \mathrm{e}^{-\kappa s} .
$$

From this, it follows that

$$
\left(\vartheta_{0}^{*}\right)^{2}=\frac{1}{T} \int_{0}^{T} \mathrm{E}\left[\sigma_{s}^{2}\right] \mathrm{d} s=\theta+\frac{\left(v_{0}-\theta\right)}{T} \int_{0}^{T} \mathrm{e}^{-\kappa s} \mathrm{~d} s=\theta+\frac{\left(v_{0}-\theta\right)\left(1-\mathrm{e}^{-\kappa T}\right)}{\kappa T} .
$$

Now consider the expression $\mathrm{E}\left[\int_{0}^{T} \Lambda_{s} \mathrm{~d} s\right]$. By definition of $\Lambda_{s}$ we have

$$
\mathrm{E}\left[\int_{0}^{T} \Lambda_{s} \mathrm{~d} s\right]=\mathrm{E}\left[\int_{0}^{T}\left(\int_{s}^{T} \mathrm{E}\left[D_{s} \sigma_{r}^{2} \mid \mathcal{F}_{s}\right] \mathrm{d} r\right) \sigma_{s} \mathrm{~d} s\right]
$$

Lemma 5.3. Assume that $2 \kappa \theta \geq v^{2}$, then

$$
\mathrm{E}\left[D_{s} \sigma_{r}^{2} \mid \mathcal{F}_{s}\right]=v \mathrm{e}^{-\kappa(r-s)} \sqrt{v_{s}} .
$$

Proof. Note that it follows, from Corollary 4.2 and the Clark-Ocone formula [12, Proposition 1.5.3], that

$$
v_{r}=\sigma_{r}^{2}=\mathrm{E}\left[\sigma_{r}^{2}\right]+\int_{0}^{r} \mathrm{E}\left[D_{s} \sigma_{r}^{2} \mid \mathcal{F}_{s}\right] \mathrm{d} W_{s} .
$$

On the other hand, consider the process defined by the stochastic integral equation

$$
\tilde{v}_{r}=\theta+\left(v_{0}-\theta\right) \mathrm{e}^{-\kappa r}+v \int_{0}^{r} \mathrm{e}^{-\kappa(r-s)} \sqrt{\tilde{v}_{s}} \mathrm{~d} W_{s} .
$$

Taking differentials of $\tilde{v}_{r}$ leads to

$$
\begin{aligned}
\mathrm{d} \tilde{v}_{r} & =-\kappa\left(\left(v_{0}-\theta\right) \mathrm{e}^{-\kappa r}+v \int_{0}^{r} \mathrm{e}^{-\kappa(r-s)} \sqrt{\tilde{v}_{s}} \mathrm{~d} W_{s}\right) \mathrm{d} r+\nu \sqrt{\tilde{v}_{r}} \mathrm{~d} W_{r} \\
& =\kappa\left(\theta-\tilde{v}_{r}\right)+\nu \sqrt{\tilde{v}_{r}} \mathrm{~d} W_{r} .
\end{aligned}
$$

Therefore, we see that $\tilde{v}_{r}$ has the same differential as $v_{r}$ and since $\mathrm{E}\left[\tilde{v}_{r}\right]=\mathrm{E}\left[v_{r}\right]$, we have 
$\tilde{v}_{r}=v_{r}$. This leads to

$$
\mathrm{E}\left[\sigma_{r}^{2}\right]+\int_{0}^{r} \mathrm{E}\left[D_{s} \sigma_{r}^{2} \mid \mathcal{F}_{s}\right] \mathrm{d} W_{s}=\mathrm{E}\left[v_{r}\right]+v \int_{0}^{r} \mathrm{e}^{-\kappa(r-s)} \sqrt{v_{s}} \mathrm{~d} W_{s},
$$

and since $\mathrm{E}\left[\sigma_{r}^{2}\right]=\mathrm{E}\left[v_{r}\right]$, Lemma 5.3 follows from the uniqueness of this representation.

By the definition of $\Lambda_{s}$ and application of Lemma 5.3, we now obtain

$$
\begin{aligned}
\mathrm{E}\left[\int_{0}^{T} \Lambda_{s} \mathrm{~d} s\right] & =\mathrm{E}\left[\int_{0}^{T}\left(\int_{s}^{T} \mathrm{E}\left[D_{s} \sigma_{r}^{2} \mid \mathcal{F}_{s}\right] \mathrm{d} r\right) \sigma_{s} \mathrm{~d} s\right] \\
& =v \mathrm{E}\left[\int_{0}^{T}\left(\int_{s}^{T} \mathrm{e}^{-\kappa(r-s)} \mathrm{d} r\right) \sigma_{s}^{2} \mathrm{~d} s\right] \\
& =v \int_{0}^{T}\left(\int_{s}^{T} \mathrm{e}^{-\kappa(r-s)} \mathrm{d} r\right) \mathrm{E}\left[\sigma_{s}^{2}\right] \mathrm{d} s \\
& =v \int_{0}^{T}\left(\int_{s}^{T} \mathrm{e}^{-\kappa(r-s)} \mathrm{d} r\right)\left(\theta+\left(v_{0}-\theta\right) \mathrm{e}^{-\kappa s}\right) \mathrm{d} s .
\end{aligned}
$$

These integrals can be evaluated easily, and we obtain

$$
\mathrm{E}\left[\int_{0}^{T} \Lambda_{s} \mathrm{~d} s\right]=\frac{v}{\kappa^{2}}\left(\theta(\kappa-2)+v_{0}+\mathrm{e}^{-\kappa T}\left(\kappa T\left(\theta-v_{0}\right)+2 \theta-v_{0}\right)\right) .
$$

With these explicit expressions for $\vartheta_{0}^{*}$ and $\mathrm{E}\left[\int_{0}^{T} \Lambda_{s} \mathrm{~d} s\right]$, (5.6), which by the previous discussion approximates the effect of correlation on option prices, becomes semi-explicit, depending on the corresponding option valuation formula in the Black-Scholes model. If this value does not admit an explicit expression, we can use Monte Carlo methods in order to compute it.

\section{A numerical example}

For a standard European call option, the expression $H$ in (5.6) is given by

$$
\frac{1}{2} H(0, x, \sigma)=\frac{\mathrm{e}^{x}}{2 \sigma \sqrt{2 \pi T}} \exp \left(-\frac{d_{1}^{2}}{2}\right)\left(1-\frac{d_{1}}{\sigma \sqrt{T}}\right),
$$

where

$$
d_{1}=\frac{x-\ln K+r T}{\sigma \sqrt{T}}+\frac{\sigma \sqrt{T}}{2} .
$$

The effect of correlation on option prices using our approximation can then be obtained in explicit form by substituting the corresponding expressions above into (5.6). The following figures illustrate the accuracy of our approximation.

Figure 1 exemplifies the percentage error of approximation (5.7) relative to the option price computed using a standard analytic Heston pricer for European calls, such as is available at http://kluge.in-chemnitz.de/tools/pricer/. The model parameters have been chosen as $\kappa=8$, $\theta=0.04, v=0.1, r=0.0953, \sigma_{0}^{2}=0.0225, S_{0}=100, T=0.1$, and $K=100$. These parameters satisfy the strong coefficient condition $2 \kappa \theta \geq 3 v^{2}$ in Proposition 5.3. The figure shows, in particular, that in percentage terms the larger part of the error is produced by replacing $\mathrm{E}\left[\mathrm{BS}\left(t, X_{t}, \vartheta_{t}\right) \mid \mathcal{F}_{t}\right]$ in Proposition 5.1 with $\mathrm{BS}\left(t, X_{t} ; \vartheta_{t}^{*}\right)$ in Proposition 5.3, while the error 


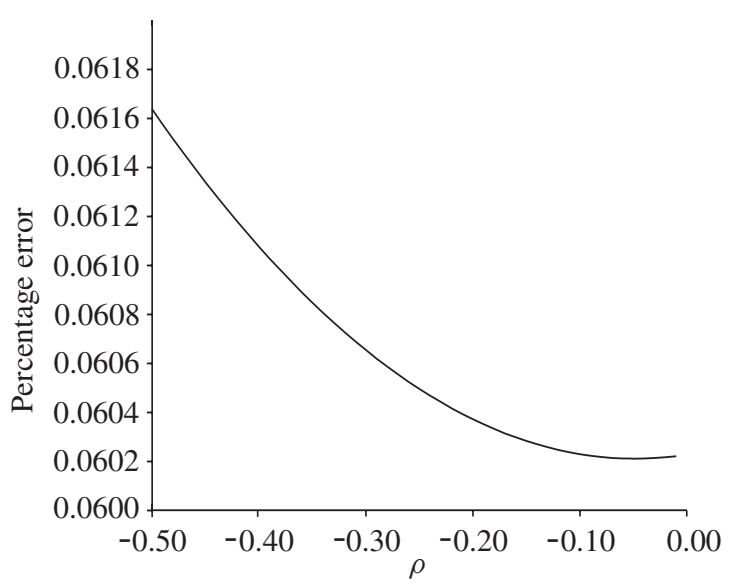

FIGURE 1: Error of approximation from Proposition 5.3 as a function of $\rho$.

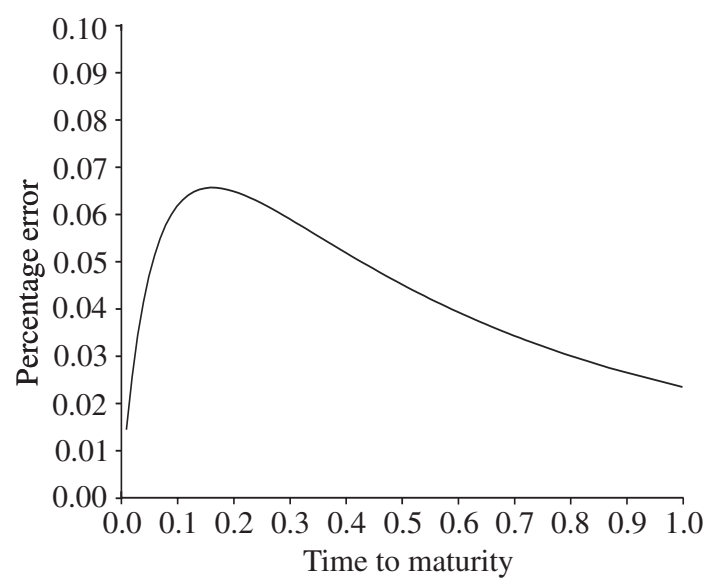

FiguRE 2: Error of approximation from Proposition 5.3 as a function of $T-t$.

contributed by our approximation of the correlation effect decreases to 0 as the correlation $\rho$ decreases to 0 .

Figure 2 illuminates the dependence of the accuracy of our approximation on time to maturity $T-t$. Displayed is the percentage error of approximation (5.7) as a function of time to maturity. The parameters in this figure are identical to the ones used for Figure 1. We observe convergence for small times to maturity, and observe that the percentage error appears to flatten out for large times to maturity. We note that the maximum percentage error found is around $0.065 \%$ and obtained at around $T-t=0.15$.

Finally, Figure 3 is obtained from Figure 2 by changing the key parameters to $\kappa=2$, $\theta=0.015$, and $v=0.2$, which correspond to the situation $2 \kappa \theta<v^{2}$, violating the strong coefficient condition in Proposition 5.3. It can be observed that in this case the percentage error is significantly higher, convergence for small times to maturity is still present, and, for large times to maturity, the flattening is clearly slower. A study of the precise behavior for large times to maturity is the subject of future research. 


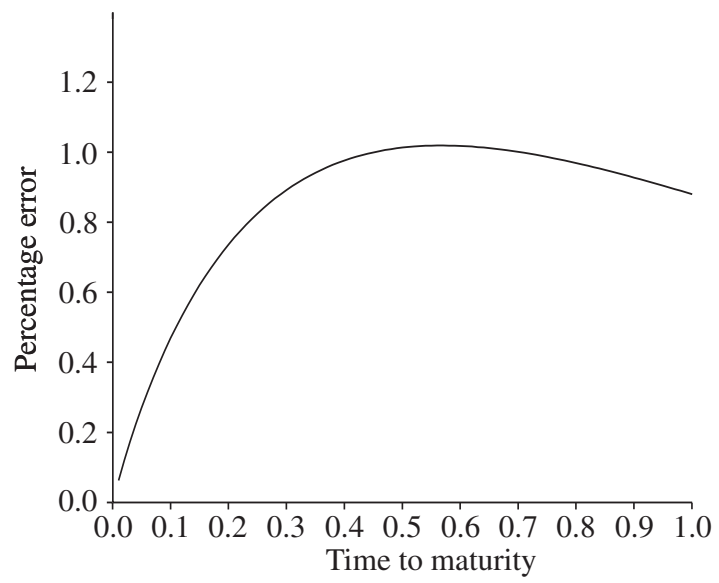

FIGURE 3: Error of approximation from Proposition 5.3 as a function of $T-t$ with $2 \kappa \theta<3 v^{2}$.

\section{Conclusions}

We have proved that under the usual coefficient condition $2 \kappa \theta \geq v^{2}$, the Heston stochastic volatility $v_{t}$ as well as its square root $\sigma_{t}$ are Malliavin differentiable and we have given compact formulae for their derivatives. Under stricter conditions on the coefficients we have shown that the second Malliavin derivatives also exist. These two results are key results in so far as they open the door for applications of Malliavin calculus in the framework of the Heston stochastic volatility model. We have discussed an explicit application by deriving an approximate option pricing formula for the Heston model, which is extremely accurate and easy to compute. Furthermore, we derived analytic expressions which control the error of this approximation.

\section{Acknowledgements}

The authors gratefully acknowledges support from grants MEC FEDER MTM2006 06427 , SEJ2006-13537, and the research grant 'Dependable adaptive systems and mathematical modeling', Rheinland-Pfalz Excellence Cluster. Furthermore, the second author would like to thank Ralf Korn (University of Kaiserslautern) and Olaf Menkens (Dublin City University) for many suggestions, fruitful discussions, and useful hints. The results of this article have been presented by the second author at the DMV-Conference in Bonn 2006 and at the '2nd Workshop on Stochastic Equations' in Jena 2006. The second author wishes to thank the organizers Professor Schael, Professor Riedel, as well as Professor Engelbert, and gratefully acknowledges travel grants from the DFG and the Royal Society. Both authors wish to thank two anonymous referees for carefully reading an earlier version of this article and giving very helpful comments, ideas, and advice, which we used to improve this article.

\section{References}

[1] Alòs, E. (2006). An extension of the Hull and White formula with applications to option pricing approximation. Finance Stoch. 10, 353-365.

[2] Alòs, E. And Nualart, D. (1998). An extension of Itô's formula for anticipating processes. J. Theoret. Prob. 11, 493-514.

[3] Alòs, E., LEON, J. A. AND Vives, J. (2007). On the shorttime behavior of the implied volatility for jump-diffusion models with stochastic volatility. Finance Stoch. 11, 571-598. 
[4] Borodin, A. N. And Salminen, P. (2002). Handbook of Brownian Motion-Facts and Formulae, 2nd edn. Birkhäuser, Basel.

[5] Bossy, M. D. A. (2004). An efficient discretization scheme for one dimensional SDE's with a diffusion coefficient function of the form $|x|^{\alpha}, \alpha \in[1 / 2,1)$. Res. Rep. 5396 INRIA.

[6] Detemple, J., Garcia, R. and Rindisbacher, M. (2005). Representation formulas for Malliavin derivatives of diffusion processes. Finance Stoch. 9, 349-367.

[7] Geman, H. and Yor, M. (1993). Bessel Processes, Asian Options and perpetuities. Math. Finance 3, 349-375.

[8] Heston, S. L. (1993). A closed-form solution for options with stochastic volatility with applications to bond and currency options. Rev. Financial Studies 6, 327-343.

[9] Hull, J. AND White, A. (1987). The pricing of options on assets with stochastic volatilities. J. Finance 42, 281-300.

[10] Jeanblanc, M. (2006). Cours de calcul stochastique. Master 2IF EVRY. Lecture Notes, University of Évry. Available at http://www.maths.univ-evry.fr/pages_perso/jeanblanc/.

[11] Karatzas, I. and Shreve, S.-E. (1988). Brownian Motion and Stochastic Calculus (Graduate Texts Math. 113). Springer, New York.

[12] Nualart, D. (1995). The Malliavin Calculus and Related Topics. Springer, New York.

[13] Yor, M. (1992). Some Aspects of Brownian Motion, Part 1, Some Special Functionals. Birkhäuser, Basel. 